\title{
OPEN Efficient table-top dual-wavelength beamline for ultrafast transient absorption spectroscopy in the soft $\mathrm{X}$-ray region
}

\author{
Lou Barreau ${ }^{1,2,5}$, Andrew D. Ross ${ }^{1,2,5}$, Samay Garg ${ }^{1}$, Peter M. Kraus ${ }^{1,4}$, Daniel M. Neumark ${ }^{1,2}$ \& \\ Stephen R. Leone $\mathrm{e}^{1,2,3^{*}}$
}

\begin{abstract}
We present a table-top beamline providing a soft $\mathrm{X}$-ray supercontinuum extending up to $370 \mathrm{eV}$ from high-order harmonic generation with sub-13 fs $1300 \mathrm{~nm}$ driving pulses and simultaneous production of sub- $5 \mathrm{fs}$ pulses centered at $800 \mathrm{~nm}$. Optimization of high harmonic generation in a long and dense gas medium yields a photon flux of $\sim 1.4 \times 10^{6}$ photons $/ \mathrm{s} / 1 \%$ bandwidth at $300 \mathrm{eV}$. The temporal resolution of X-ray transient absorption experiments with this beamline is measured to be $11 \mathrm{fs}$ for 800 $\mathrm{nm}$ excitation. This dual-wavelength approach, combined with high flux and high spectral and temporal resolution soft X-ray absorption spectroscopy, is a new route to the study of ultrafast electronic dynamics in carbon-containing molecules and materials at the carbon K-edge.
\end{abstract}

\begin{abstract}
Transient absorption spectroscopy with high-order harmonic generation (HHG) sources is now established as a powerful technique to probe ultrafast dynamics in atoms ${ }^{1-3}$, molecules $^{4-6}$ and solids ${ }^{7-10}$ (see e.g. ref. ${ }^{11}$ for a review). Being an all-optical method, in which the timing is done first and the spectral resolution separately, the time and energy resolution are independent and not subject to an uncertainty relationship. It can therefore combine the attosecond temporal capabilities of HHG sources with the atomic-specificity and the chemical environment sensitivity of X-ray spectroscopy. In X-ray absorption spectroscopy, intense absorption features are located around the so-called atomic edges. Many of the heavier elements show spectroscopic signatures in the extreme ultra-violet region (XUV, 10-100 eV) corresponding to $\mathrm{L}_{2,3}, \mathrm{M}_{4,5}$ or $\mathrm{N}_{4,5}$ edges. However, several key elements are not accessible in this spectral domain, including sulfur $\left(\mathrm{L}_{2,3}\right.$-edge at $163 \mathrm{eV}$ ), carbon (K-edge at 284 $\mathrm{eV})$, nitrogen (K-edge at $410 \mathrm{eV}$ ) and oxygen (K-edge at $540 \mathrm{eV}$ ), that are present in the majority of organic and biochemically-relevant molecules and materials. Recent experimental efforts (see e.g. ref. ${ }^{12}$ for a review) have thus focused on increasing the photon energy available from table-top X-ray sources, to reach the soft X-ray domain (SXR, 100-1000 eV). This is achieved by taking advantage of the scaling of the cutoff energy in the HHG process with intensity $I$ and wavelength $\lambda$ of the driving laser field as $E_{\text {cutoff }} \propto I \lambda^{2}\left(\right.$ ref. $\left.{ }^{13}\right)$. While the first SXR photons produced with a HHG source utilized high intensity $800 \mathrm{~nm}$ driving wavelength in a strong ionization regime ${ }^{14,15}$, the strategy that is now preferred is to use longer wavelength drivers in the short-wave infrared $(\mathrm{SWIR})^{12,16-18}$ and mid-infrared ${ }^{19}$.

In order to provide a spectral supercontinuum instead of discrete harmonic peaks, as well as allow the production of isolated attosecond pulses and therefore considerably improve the temporal resolution, the use of few-cycle SWIR pulses to drive SXR HHG has recently been developed. Indeed, the $9 \mathrm{fs}$ output of an optical parametric chirped pulse amplifier centered at $1600 \mathrm{~nm}$ generated a SXR continuum up to $350 \mathrm{eV}$ in neon ${ }^{20}$. By taking advantage of the polarization gating technique with $12 \mathrm{fs}, 1800 \mathrm{~nm}$ pulses, and the appropriate choice of metallic filters to compensate for the SXR dispersion ${ }^{21}$, an isolated pulse of 53 attoseconds extending above $300 \mathrm{eV}$ was measured ${ }^{22}$. Likewise, more conventional approaches relying on the post-compression of 30-50 fs idler pulses from Ti:sapphire-pumped Optical Parametric Amplifiers (OPA) produced SXR radiation supporting isolated
\end{abstract}

\footnotetext{
${ }^{1}$ Department of Chemistry, University of California, Berkeley, CA, 94720, USA. ${ }^{2}$ Chemical Sciences Division, Lawrence Berkeley National Laboratory, Berkeley, CA, 94720, USA. ${ }^{3}$ Department of Physics, University of California, Berkeley, CA, 94720, USA. "Present address: Advanced Research Center for Nanolithography (ARCNL), Science Park 106, 1098 XG, Amsterdam, The Netherlands. ${ }^{5}$ These authors contributed equally: Lou Barreau and Andrew D. Ross. *email: srl@berkeley.edu
} 


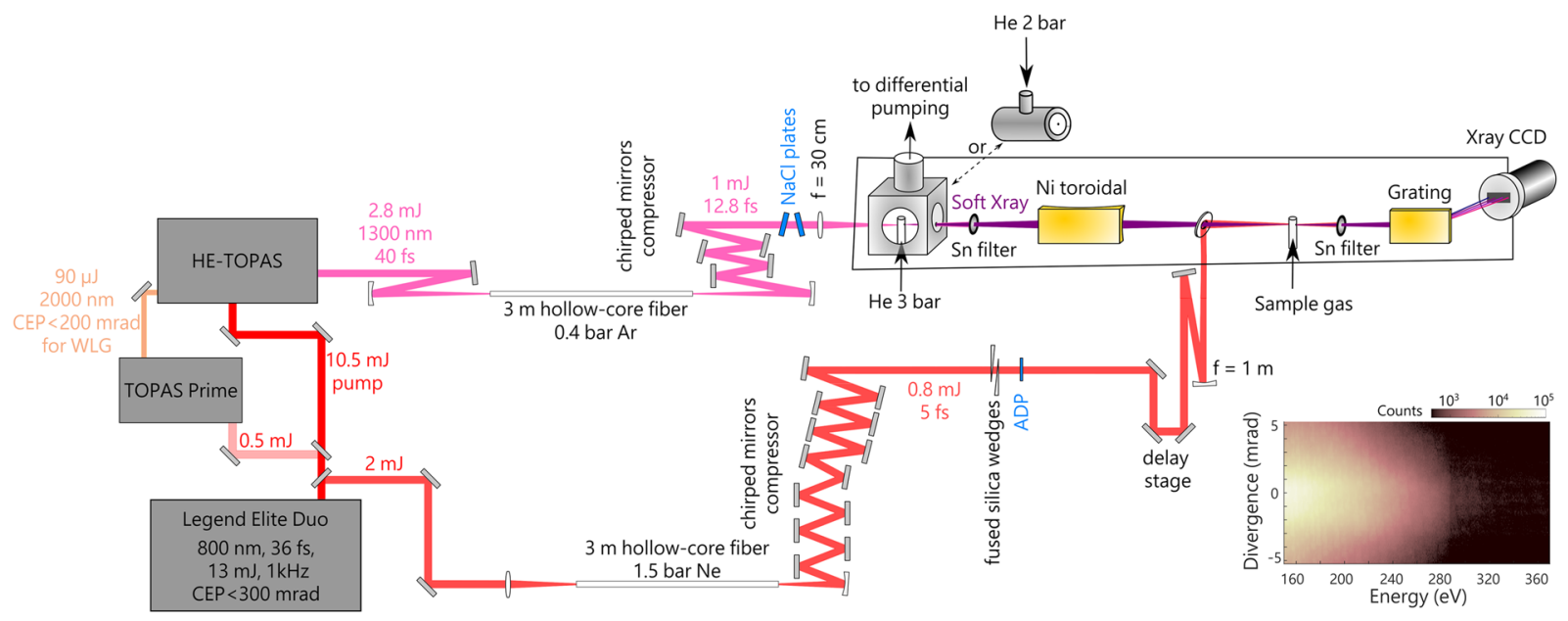

Figure 1. Summary of the beamline. Soft X-ray pulses are produced from high-order harmonic generation with few-cycle SWIR pulses in helium. Few-cycle pulses centered at $800 \mathrm{~nm}$ are simultaneously produced and are used as a pump in ultrafast transient absorption experiments. The different steps are detailed in the text. A typical X-ray camera image is shown at the bottom right. The drop in signal after $\sim 280 \mathrm{eV}$ is due to carbon contamination of the X-ray optics, causing absorption at the carbon K-edge.

attosecond pulses ${ }^{23,24}$. All of these studies use few-cycle SWIR pulses in the 1600-1850 nm range, which offer the advantages of (i) passive Carrier-Envelope Phase (CEP) stabilization ${ }^{25}$ and (ii) the use of transmission through bulk glass for post-compression ${ }^{26}$. However, compared to shorter wavelengths, they suffer unfavourable scaling of the HHG efficiency with the driving wavelength, $\propto \lambda^{-6}$ at the single-atom level ${ }^{27,28}$. In those conditions, a single absorption spectrum requires accumulation of the signal over a few minutes ${ }^{22,29}$. Reduced integration times will be decisive for the application of SXR transient absorption spectroscopy to the study of attosecond chemical dynamics in systems containing $\mathrm{S}, \mathrm{C}$ or $\mathrm{N}$ atoms ${ }^{30-32}$. Therefore, a trade-off comprising a continuous spectrum extending above the carbon K-edge with high flux and few-femtosecond temporal resolution would be desirable. Moreover, transient absorption in the XUV typically employs a fraction of energy of the few-cycle visible-near IR pulses used for HHG as a pump via strong-field $d^{4,6}$ or single-photon excitation ${ }^{5,8-10}$. Due to the smaller photon energy in the SWIR, using a similar approach for SXR transient absorption considerably reduces the pumping excitation capabilities. The availability of an intense, ultrashort, second color pulse in the visible-near infrared synchronous with the SXR-generating SWIR used as a pump would circumvent this issue. Note however that field-driven carrier motion in a semi-metal ${ }^{33}$ and tunnel ionization of a low ionization potential molecule ${ }^{34}$ with $\lambda>1600 \mathrm{~nm}$ few-cycle pulses have recently been observed.

In this work, a table-top beamline simultaneously producing few-cycle SWIR and vis-NIR pulses to be used in ultrafast pump-probe experiments in the SXR is presented. The two-cycle pulses centered at $1300 \mathrm{~nm}$ are used to drive HHG in helium, providing a continuous spectrum extending up to $370 \mathrm{eV}$, with $\sim 1.4 \times 10^{6}$ photons/s/1\% bandwidth at $300 \mathrm{eV}$. We show that the SXR pulses produced enable the measurement of high resolution absorption spectra in the 170-300 eV range within a few seconds. When used in combination with sub-5 fs vis-NIR pulses, the temporal resolution achieved in a transient absorption experiment at $250 \mathrm{eV}$ is $\sim 11 \mathrm{fs}$.

\section{Description of the table-top dual-wavelength beamline}

The beamline is summarized in Fig. 1. It is based on a $13 \mathrm{~mJ}, 800 \mathrm{~nm}, 30 \mathrm{fs}, 1 \mathrm{kHz}$ Ti:Sapphire laser (Coherent Legend Elite Duo), whose energy is split into $11 \mathrm{~mJ}$ and $2 \mathrm{~mJ}$ to produce the probe and pump pulses, respectively, used in time-resolved X-ray transient absorption experiments. This section details the simultaneous compression of SWIR (centered at $1300 \mathrm{~nm}$ or $1400 \mathrm{~nm}$ ) and visible-near infrared (centered at $800 \mathrm{~nm}$ ) pulses, and the characteristics of the soft X-ray spectrometer.

Production of few-cycle short-wave infrared pulses. Few-cycle pulses centered at $1300 \mathrm{~nm}$ in the SWIR are produced using an OPA followed by spectral broadening in a hollow-core fiber filled with a rare gas ${ }^{35}$ and compression with chirped mirrors ${ }^{36}$. The $11 \mathrm{~mJ} 800 \mathrm{~nm}$ beam is further split into $0.5 \mathrm{~mJ}$ and $10.5 \mathrm{~mJ}$ for use in a two-stage OPA, which converts $800 \mathrm{~nm}$ to SWIR $(1300 \mathrm{~nm})$. The two-stage system is obtained from Light Conversion and is designed to provide CEP stability of the signal pulses. The first stage is a low energy OPA (TOPAS Prime), pumped by $0.5 \mathrm{~mJ}$, which provides $90 \mu \mathrm{J}$ of $2 \mu \mathrm{m}$ pulses in the idler. Due to the parametric amplification process, the idler pulses are passively CEP stabilized, with a stability of $<200 \mathrm{mrad}$. They are then used as the seed for the white light generation (WLG) in a second, high energy, OPA (HE-TOPAS) pumped with the remaining $10.5 \mathrm{~mJ}$ of $800 \mathrm{~nm}$ light. This design should ensure the CEP stability of the signal pulses over the 1200-1600 nm tunable range of the OPA, regardless of the CEP stability of the pump laser. However, the following results are obtained with a CEP-averaged signal pulse.

The $2.8 \mathrm{~mJ}, 1300 \mathrm{~nm}, 40 \mathrm{fs}$ output is focused with a 2-m focusing mirror to a focal size of $\left.390 \mu \mathrm{m} \mathrm{(at} 1 / \mathrm{e}^{2}\right)$ at the entrance of a 3-m-long, 700- $\mu \mathrm{m}$-inner diameter stretched hollow-core fiber (HCF) filled with 0.4 bar of argon (Few-Cycle Inc.). The ratio of beam waist to fiber diameter was experimentally adjusted for best transmission 
Measured SHG FROG

$1300 \mathrm{~nm}$

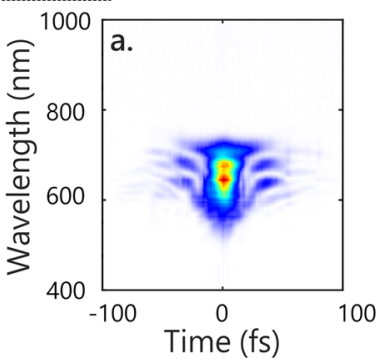

$1400 \mathrm{~nm}$

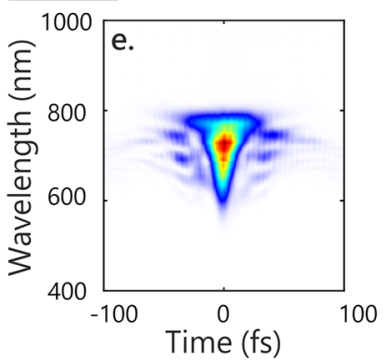

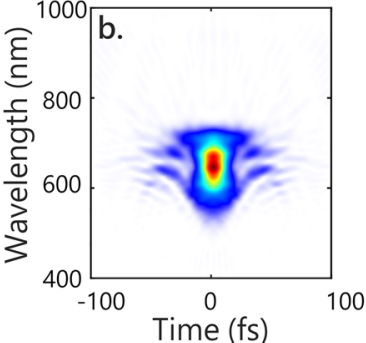

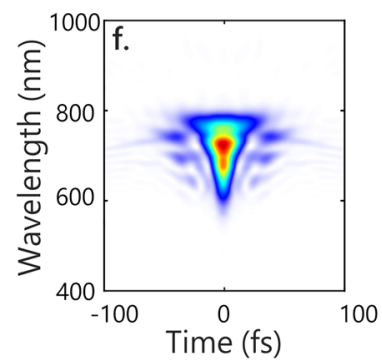

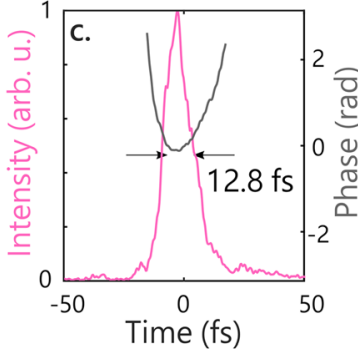
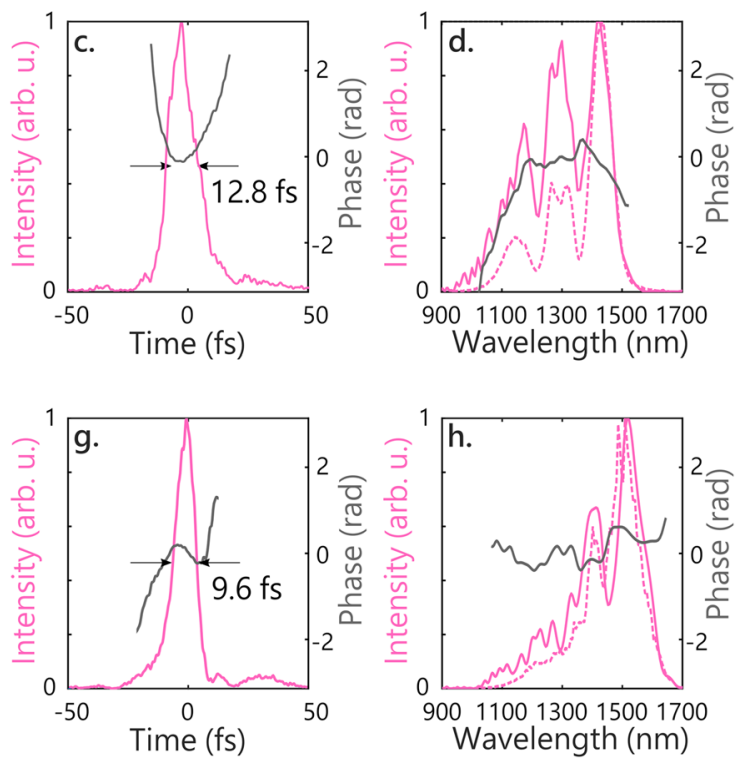

Figure 2. Characterization of the compressed SWIR pulses produced with the OPA central wavelength set at $1300 \mathrm{~nm}(\mathbf{a}-\mathbf{d})$ and $1400 \mathrm{~nm}(\mathbf{e}-\mathbf{h})$ with SHG-FROG. (a,e) Measured spectrogram. (b,f) Retrieved spectrogram. $(\mathbf{c}, \mathbf{g})$ Retrieved temporal intensity (pink) and phase (grey). (d,h) Retrieved spectral intensity (pink) and phase (grey). The dashed line is the spectrum at the output of the fiber as measured with a spectrometer (Ocean Optics FLAME). Phases are shown for intensities $>5 \%$ of the maximum intensity.

through the fiber, and it was found that this slightly smaller value (0.56) compared to the optimal value for coupling to the $\mathrm{LP}_{01}$ mode ${ }^{37}(0.64)$ yielded higher output power. After broadening through self-phase modulation, the spectrum extends from $1000 \mathrm{~nm}$ to $1500 \mathrm{~nm}$ (see the dashed line in Fig. 2d), supporting 9.3 fs pulses with up to $1.45 \mathrm{~mJ}$ per pulse. After recollimation, the pulses are compressed with a set of four ultra-broadband dispersive mirrors ( $\mathrm{PC} 1816$, Ultrafast Innovations $\mathrm{GmbH}$ ) that provide an average of $-150 \mathrm{fs}^{2}$ per reflection over the 1000-1700 $\mathrm{nm}$ spectral range. For fine-tuning of the group-delay dispersion (GDD), the beam goes through a pair of counter-rotating $2-\mathrm{mm} \mathrm{NaCl}$ windows, which have a GDD of $+47.8 \mathrm{fs}^{2} / \mathrm{mm}$ at $1300 \mathrm{~nm}$. The pulses are characterized using a home-built dispersion-free second harmonic generation Frequency-Resolved Optical Gating (SHG-FROG), whose results are shown in Fig. 2a-d. The retrieved pulse has a full width at half maximum (fwhm) in intensity of $12.8 \mathrm{fs}$, which corresponds to less than 3 optical cycles, with $80 \%$ of the energy in the main pulse. Further compression could be achieved with a better control over third-order dispersion.

The ultra-broadband dispersive mirrors are optimized for chirp compensation over a 700-nm bandwidth in the SWIR. In order to test the tunability of the HCF compressor, the central wavelength of the OPA is increased to $1400 \mathrm{~nm}$, the rest of the system remaining identical. The spectrum is broadened in 0.6 bar of argon and extends from 1050 to $1600 \mathrm{~nm}$ (Fig. 2h), with up to $1.15 \mathrm{~mJ}$ per pulse. From the SHG-FROG measurement and retrieval shown in Fig. $2 \mathrm{e}-\mathrm{g}$, the pulses are compressed down to $9.6 \mathrm{fs}$ fwhm, that is 2 cycles at $1400 \mathrm{~nm}$, with $88 \%$ of the energy in the main pulse. Thus, we see that the system allows wavelength tuning in the SWIR over a $100 \mathrm{~nm}$ range.

Simultaneous production of few-cycle visible-near IR pulses. The $2 \mathrm{~mJ}$ pulses at $800 \mathrm{~nm}$ split before the pumping of the OPA stages (see Fig. 1) are compressed to few-cycle duration using the same $\operatorname{method}^{35}$ in a second, $400-\mu \mathrm{m}$-inner diameter stretched HCF filled with 1.5 bar of neon. After propagation over two meters to compensate for the delay acquired by the SWIR pulse in the OPA stages, the beam is focused with a 2-m focusing lens to a $280 \mu \mathrm{m}$ spot size at the entrance to the fiber. At the output, the pulse energy is $0.8 \mathrm{~mJ}$ with a spectrum covering 550-950 nm (Fig. 3c). Compression is achieved with reflections on sixteen ultra-broadband dispersive mirrors (PC1332, Ultrafast Innovations $\mathrm{GmbH}$ ), transmission through fused silica wedges and finally through a 2-mm-thick Ammonium Dihydrogen Phosphate (ADP) crystal, which corrects for third-order dispersion ${ }^{38}$. The visible-near IR (vis-NIR) pulses are characterized with a d-scan module (Sphere Ultrafast Photonics) ${ }^{39}$, whose results are shown in Fig. 3. The d-scan phase retrieval algorithm indicates a pulse duration of $4.75 \mathrm{fs}$ fwhm, which is 1.1 times the Fourier limit, with $75 \%$ of the intensity in the main pulse. Compression of the vis-NIR pulses to 1.8 cycle is realized at the same time as the compression of the SWIR pulses described in the previous section. Therefore, these two pulses can be used together in a pump-probe experiment. We note that shorter pulse durations in the vis-NIR with similar pulse energy can be achieved with this $\operatorname{method}^{38}$, and could most likely be obtained here if the compression of the Ti:Sa laser could be tuned independently for pumping the OPA stages and the vis-NIR HCF compressor. 

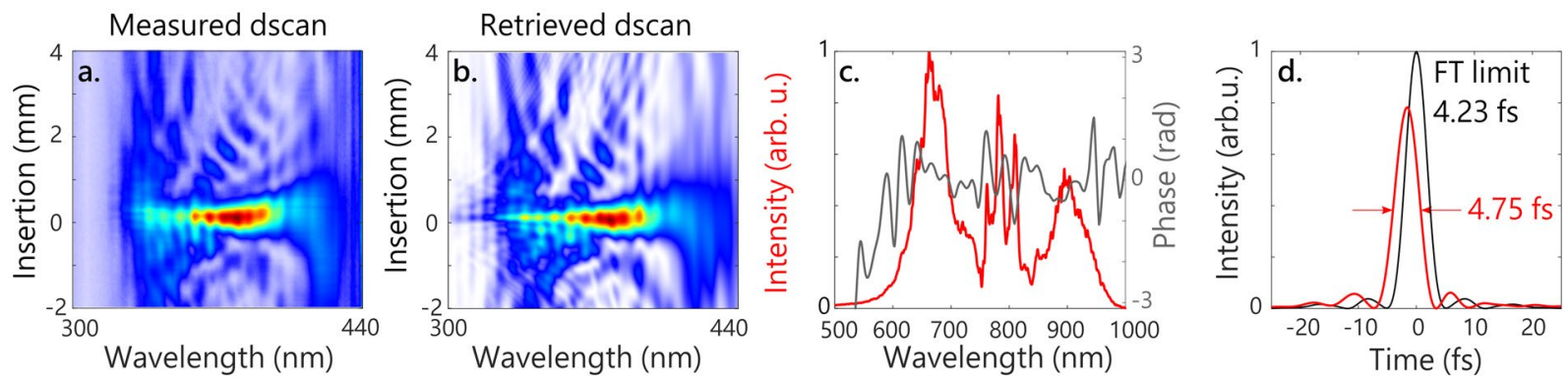

Figure 3. Characterization of the few-cycle $800 \mathrm{~nm}$ pulses used as a pump. (a) Measured dscan. (b) Retrieved dscan. (c) Measured spectrum (red) and retrieved spectral phase (grey) of the compressed pulses. (d) Retrieved temporal intensity (red) and Fourier-limited pulse (black).
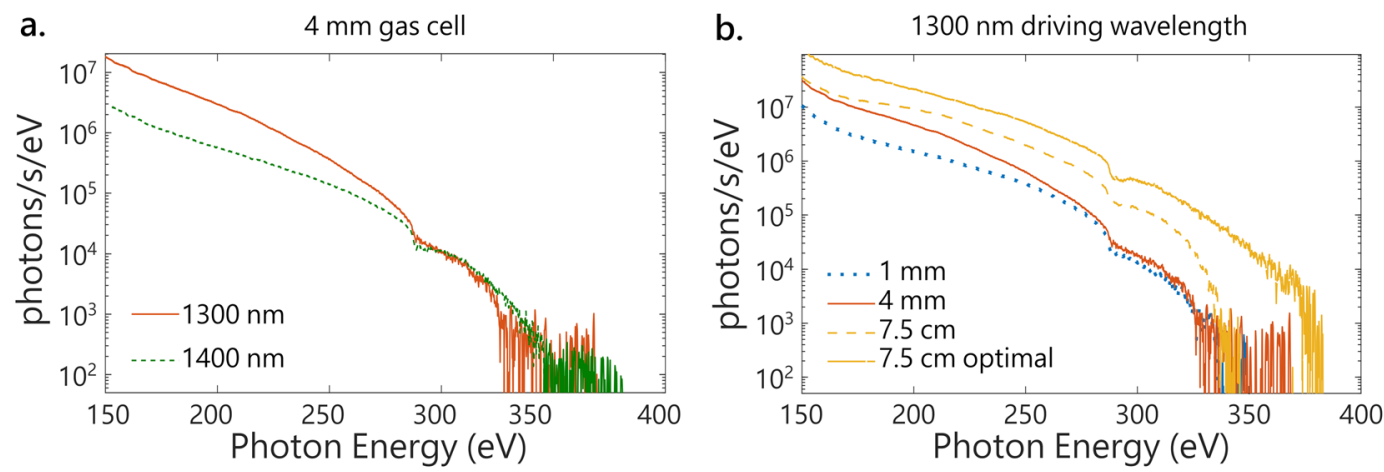

Figure 4. Optimization of the flux at $300 \mathrm{eV}$ with the driving wavelength and the gas cell length. (a) Soft X-ray high-order harmonic spectra generated in helium with few-cycles pulses with the OPA central wavelength set at $1300 \mathrm{~nm}$ (red, solid) and $1400 \mathrm{~nm}$ (green, dashed) in a $4 \mathrm{~mm}$ gas cell. (b) Soft X-ray high-order harmonic spectra generated in helium with few-cycles pulses with the OPA central wavelength set at $1300 \mathrm{~nm}$ in $1 \mathrm{~mm}$ (blue, dotted), $4 \mathrm{~mm}$ (red, solid) and $7.5 \mathrm{~cm}$ (yellow, dashed) length gas cells. Further optimization of the SWIR HCF gas pressure for HHG with the $7.5 \mathrm{~cm}$ cell yields the optimal solid yellow curve.

Soft X-ray spectrometer. The soft X-ray (SXR) pulses are produced via high-order harmonic generation by focusing $0.7 \mathrm{~mJ}$ of the few-cycle SWIR pulses with a $f=30 \mathrm{~cm}$ lens down to a $80 \mu \mathrm{m}\left(\right.$ at $\left.1 / e^{2}\right)$ diameter focus $\left(\sim 2 \times 10^{15} \mathrm{~W} / \mathrm{cm}^{2}\right)$ into a 4 -mm-long gas cell continuously flowing with helium at a backing pressure of 2.8 bar (see Fig. 1). The use of a lens is unusual with few-cycle driving pulses ${ }^{5,22-24,40}$; however, the near 0 GDD of fused silica at $1300 \mathrm{~nm}\left(-2.4 \mathrm{fs}^{2} / \mathrm{mm}\right)$ provides minimal pulse lengthening from chirp and geometrical sources ${ }^{41}$. The gas cell is surrounded by a $7 \mathrm{~cm}$ edge-length cube for differential pumping, sealed along the propagation axis with cellophane tape through which holes are laser-produced. The cube is evacuated by a roots blower with $900 \mathrm{~m}^{3} / \mathrm{h}$ pumping speed, such that the pressure inside is calculated to be $\sim 1$ Torr and is measured to be $\sim 1 \times 10^{-3}$ Torr in the surrounding chamber. After HHG, the SWIR light is filtered out with a metallic foil ( $\mathrm{Zr}, \mathrm{Sn}, \mathrm{Ag}$, Ti or Al can be used depending on the spectral region of interest). The SXR is focused by a Ni-coated toroidal mirror at $4^{\circ}$ grazing angle into the sample, which can be a gas cell or a solid membrane. Changes between different samples can be easily achieved with motorized stages in the two dimensions perpendicular to the propagation axis. The spectrum is dispersed by an aberration-corrected concave grating and imaged onto an X-ray CCD camera (Greateyes, GE 2048512 BI UV1). The camera is movable along the grating focal plane to fully capture the SXR spectrum. Depending on the spectral region of interest, two different gratings can be used, optimized for 5-25 nm (50-247 eV, Hitachi 001-0660) or 1-6 nm (207-1239 eV, Hitachi 001-0659), referred to as the low- and high-energy gratings in the following. The grating rotation and camera position can be adjusted to optimize the spectral resolution. The resolution of the spectrometer has been determined using the $\operatorname{Ar} 2 \mathrm{p}_{3 / 2}^{-1} 4 \mathrm{~s}$ absorption line ${ }^{42}$ to be 325 and $190 \mathrm{meV}$ fwhm at $244.39 \mathrm{eV}$, corresponding to $\Delta E / E=1.3 \times 10^{-3}$ and $\Delta E / E=7.8 \times 10^{-4}$ for the two gratings, respectively. While the resolution of the high-energy grating is higher, its increased groove density results in a lower efficiency by a factor of $\sim 3$. Unless otherwise mentioned, all the spectra shown in the following are measured using the low-energy grating.

\section{Optimization of the photon flux at the carbon K-edge}

Driving wavelength. Here we study how the high-harmonic flux in the cutoff region differ for two few-cycle driving pulses of different wavelengths in the SWIR. Indeed, at the single-atom level, the HHG efficiency scales with $\lambda^{-6}$ (refs. ${ }^{27,28}$ ). However, this goes together with an increase in the cutoff photon energy as $\lambda^{2}$ (ref. ${ }^{13}$ ), and calculations have shown that the atomic response can be macroscopically overcome under certain 


\begin{tabular}{|l|l|l|l|}
\hline Condition & $\begin{array}{l}\text { Photons/s in 1\% } \\
\text { BW at 300 eV }\end{array}$ & $\begin{array}{l}\text { Photons/s in 10\% } \\
\text { BW at 300 eV }\end{array}$ & $\begin{array}{l}\text { Photons/s } \\
\text { in 1\% BW } \\
\text { at } \mathbf{2 0 0} \mathbf{~ e V}\end{array}$ \\
\hline $1400 \mathrm{~nm}, 4 \mathrm{~mm}$ cell & $3.4 \times 10^{4}$ & $3.3 \times 10^{5}$ & $1.18 \times 10^{6}$ \\
\hline $1300 \mathrm{~nm}, 4 \mathrm{~mm}$ cell & $3.4 \times 10^{4}$ & $3.6 \times 10^{5}$ & $6.4 \times 10^{6}$ \\
\hline $1300 \mathrm{~nm}, 1 \mathrm{~mm}$ cell & $2.5 \times 10^{4}$ & $2.7 \times 10^{5}$ & $2.0 \times 10^{6}$ \\
\hline $1300 \mathrm{~nm}$, semi-infinite $7.5 \mathrm{~cm}$ cell & $2.5 \times 10^{5}$ & $2.4 \times 10^{6}$ & $1.2 \times 10^{7}$ \\
\hline $1300 \mathrm{~nm}$, semi-infinite, optimal & $1.4 \times 10^{6}$ & $1.3 \times 10^{7}$ & $4.5 \times 10^{7}$ \\
\hline $1850 \mathrm{~nm}, 12 \mathrm{fs}$ in $\mathrm{Ne}^{23}$ & & $2.8 \pm 0.1 \times 10^{7}$ & \\
\hline $1850 \mathrm{~nm}, 12 \mathrm{fs}$ in $\mathrm{He}^{23}$ & & $1.8 \pm 0.1 \times 10^{6}$ & \\
\hline $1800 \mathrm{~nm}, 12 \mathrm{fs}$ in $\mathrm{Ne}^{24}$ & $3.0 \pm 0.8 \times 10^{6}$ & & \\
\hline $1800 \mathrm{~nm}, 12 \mathrm{fs}$ in $\mathrm{He}^{24}$ & $1.6 \pm 0.4 \times 10^{5}$ & & \\
\hline
\end{tabular}

Table 1. Comparison of photon fluxes in the soft X-ray in existing beamlines using HHG driven by few-cycle SWIR pulses.

phase-matching conditions ${ }^{17}$. In the previous section, the tunability of the few-cycle SWIR source was demonstrated. We therefore compare the SXR spectra produced when driving the HHG in the $4 \mathrm{~mm}$ gas cell with the $1300 \mathrm{~nm}$ and $1400 \mathrm{~nm}$ pulses characterized in Fig. 2. The HHG spectra produced in these conditions are shown in Fig. 4a. From the measured spectra, the photon fluxes are calculated at the source using the known quantum efficiency of the CCD camera and number of electrons generated per X-ray photon of a given energy, as well as tabulated toroidal mirror reflectivity, metallic filter transmission, and grating efficiency. At both wavelengths, the spectra are continuous and extend from below $150 \mathrm{eV}$ (not shown in Fig. 4 because of the limited size of the camera chip) to above $300 \mathrm{eV}$. As expected, the maximum photon energy produced with few-cycle pulses centered at $1400 \mathrm{~nm}$ is higher compared to $1300 \mathrm{~nm}$. The photon flux is 2 to 10 times larger at the $1300 \mathrm{~nm}$ driving wavelength over the 150-280 eV spectral range. However, in the carbon K-edge region above $280 \mathrm{eV}$, the two drivers produce similar high harmonic fluxes. The measured photon fluxes, in photons/s in $1 \%$ and $10 \%$ bandwidth at $300 \mathrm{eV}$, are summarized in Table 1 and compared to literature values for SXR HHG sources driven by few-cycle SWIR pulses.

HHG gas cell length. The question of macroscopic effects in HHG with SWIR pulses has been the subject of recent experimental and theoretical work ${ }^{16-18,23}$. Here we compare the SXR flux obtained in helium with few-cycle $1300 \mathrm{~nm}$ pulses in continuously flowed gas cells of different lengths. The spectrum obtained in a $4 \mathrm{~mm}$ gas cell described previously is reproduced in red, solid in Fig. 4b. Johnson et al. showed that, in the so-called overdriven regime, plasma defocusing effects strongly reshape the HHG driving pulse, already in the tails of the pressure distribution around the gas cell ${ }^{24}$. In order to limit these effects, a shorter gas cell of $1 \mathrm{~mm}$ length $(1 \mathrm{~mm}$ outer diameter and $500 \mu \mathrm{m}$ inner diameter) is used in place of the $4 \mathrm{~mm}$ one described before. The corresponding HHG spectrum, generated in 4 bar of helium, is shown in blue on Fig. $4 \mathrm{~b}$. No increase of the cutoff is observed, and the flux at $300 \mathrm{eV}$ is $2 \mathrm{x}$ less than with a longer interaction medium (Table 1).

Since the absorption length at $300 \mathrm{eV}$ of 2 bar of helium is $\sim 1 \mathrm{~cm}$, a longer HHG medium was also tried ${ }^{43}$. SXR pulses are generated in a $7.5 \mathrm{~cm}$ long tube, sealed at the entrance with a $500 \mu \mathrm{m}$ thick window and with a teflon foil through which a hole is produced with the laser at the end. The focus of the SWIR is towards the exit of the cell, therefore being qualified as semi-infinite ${ }^{34,44}$. No nonlinear effects in the entrance window were observed at the working intensities. A reduced $\sim 2$ bar helium pressure is required to optimize HHG in the semi-infinite gas cell, so differential pumping is not necessary. Instead, the roots blower pump is used to back the turbomolecular pump attached to the HHG chamber, where the pressure is measured to be $\sim 10^{-3}$ Torr in these conditions. As shown in dashed yellow in Fig. 4b, this gas cell produces an order of magnitude more photon flux at $300 \mathrm{eV}$, with the maximum achievable photon energy remaining identical. Therefore, the semi-infinite gas cell is used in the following experiments. Following this observation, the other experimental parameters were optimized to maximize the SXR flux. Broadening the SWIR pulse in 0.7 bar of argon, instead of 0.4 bar previously, produces the solid yellow spectrum visible on Fig. $4 \mathrm{~b}$, with $1.4 \times 10^{6}$ photons $/ \mathrm{s}$ in $1 \%$ bandwidth at $300 \mathrm{eV}$ (see Table 1 ). The results call for further investigations of the role of ionization in HHG with few-cycle pulses in a semi-infinite gas cell.

We note that continuous SXR spectra are obtained in all the reported cases, but this observation is not proof of the production of isolated attosecond pulses - these spectra are measured with random CEP and the lack of CEP stability can cause random phase shifts in the HHG spectra that then appear as a continuum ${ }^{45}$. The semi-infinite gas cell yields more photon flux around the carbon K-edge, but is probably less suited for the production of isolated attosecond pulses. However, few-fs SXR pulses at the carbon K-edge (combined with a few-fs pump) would be able to probe ultrafast dynamics on the few tens of femtoseconds timescale, such as passage through conical intersections in organic molecules. Temporal characterization of the SXR pulses ${ }^{22,46}$ is needed, in particular in the long gas cell case, but this is out of the scope of the current paper. However, the temporal resolution of the beamline is estimated from a transient absorption experiment in the following section. It is limited by the durations of the vis-NIR pulse (see Fig. 3), the SXR probe, and the optical path fluctuation of the $\sim 15 \mathrm{~m}$ long interferometer. The delay jitter has been measured using a balanced optical cross-correlator ${ }^{47}$ to be $2 \mathrm{fs} r m s$ over $10 \mathrm{~s}$. 
a.

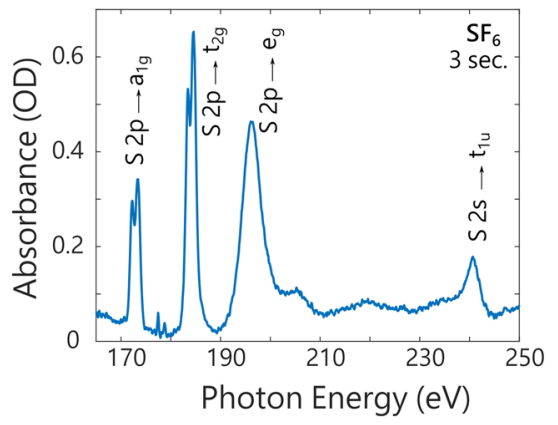

b.

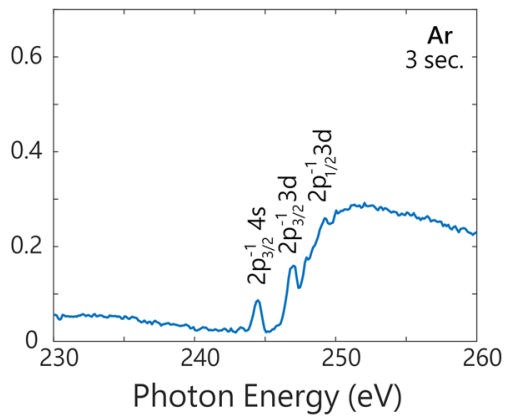

C.

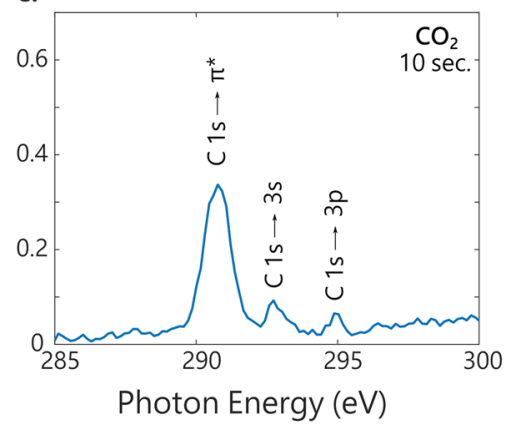

Figure 5. X-ray near-edge absorption spectra measured with the SXR HHG source in (a) $\mathrm{SF}_{6},(\mathbf{b}) \operatorname{Ar}$ and (c) $\mathrm{CO}_{2}$.

\begin{abstract}
Absorption spectroscopy in the soft X-ray Measurement of absorption spectra with the high-harmonic source. In order to demonstrate the suitability of the SXR HHG source, X-ray absorption spectra are typically measured ${ }^{22,24,40,48-51}$. As seen on Fig. 1, a gas cell can be inserted in the SXR beam path at the focus of the toroidal mirror. This allows the measurement of spectra such as shown in Fig. 5, where the semi-infinite gas cell and $1300 \mathrm{~nm}$ pulses are used for HHG (dashed yellow spectrum on Fig. 4b). The absorbance, in optical densities (OD), is defined as $A=-\log _{10} \frac{I}{I_{0}}$, where $I$ and $I_{0}$ are the background-corrected spectra measured with and without the sample, respectively. The high flux of our beamline and the continuous, broad SXR spectrum allow the measurement of absorption spectra in a few seconds only, without scanning the photon energy. The absorption spectrum of $\mathrm{SF}_{6}$ around the sulfur $\mathrm{L}_{2,3}$ and $\mathrm{L}_{1}$ edges displayed in Fig. $5 a$ is acquired in 6 seconds ( 3 seconds with and without the sample). The spin-orbit split doublet corresponding to the excitation of a S $2 p$ electron to the unoccupied $a_{1 g}$ molecular orbital is well-resolved, as well as a series of excitations to molecular Rydberg states around $180 \mathrm{eV}^{52}$. Above the $\mathrm{S} 2 p^{-1}$ ionization threshold, excitation to quasi-bound states $t_{2 g}$ and $e_{g}$ is visible, as well as the weaker transition of the $S 2 s$ electron to a $t_{1 u}$ molecular orbital around $240 \mathrm{eV}$. The short acquisition times and the high spectral resolution should allow the observation of ultrafast dynamics in the $\mathrm{SF}_{6}$ molecule ${ }^{53}$. Similarly, absorption spectra of argon around the $\mathrm{L}_{2,3}$ edge around $250 \mathrm{eV}$ are obtained in 6 seconds (Fig. 5b). At the spectral resolution of $325 \mathrm{meV}$, three peaks in the series converging to the $\operatorname{Ar} 2 p^{-1}$ threshold can be resolved ${ }^{29,42}$. Despite the lower flux at higher photon energies (see Fig. 4 and Table 1), the absorption spectrum of $\mathrm{CO}_{2}$ around the carbon K-edge can also be measured, this time in 20 seconds ( 10 seconds with and without the sample). Three main features of the spectrum can be identified, including the main C $1 s$ to $\pi^{*}$ transition $^{54}$ (Fig. 5c).
\end{abstract}

Ultrafast transient absorption in argon near the $L_{2,3}$ edge. In order to realize pump-probe experiments, the sub- 5 fs vis-NIR pulse is time-delayed and focused in the sample gas cell with a concave mirror and recombined with the SXR on a hole mirror (see Fig. 1). The optical light is filtered by a metallic foil before the SXR spectrometer. A representative transient absorption experiment is performed in argon, using SXR produced with the $7.5 \mathrm{~cm}$ long HHG gas cell filled with 2 bar of helium. The delay-dependent absorption spectra are shown in Fig. 6a. At each delay point $\tau$, the absorbance $A$ is calculated as $A(\tau)=-\log _{10} \frac{I(\tau)}{I_{0}(\tau)}$, where the reference spectrum $I_{0}$ is reconstructed by using a Fourier low-pass filter directly on the measured signal $I(\tau)$. This procedure allows for removal of the intensity fluctuations of the SXR spectra and is well-suited in the case of narrow absorption lines $^{3}$. In Fig. $6 \mathrm{a}, A$ then represents the absorption with respect to the continuum absorption background. Here, the SXR excites $2 p$ core-excited states of argon (Fig. $5 b$ ). In the presence of the vis-NIR pulse, with a polarization parallel to the SXR polarization and intensity of $\sim 3 \times 10^{13} \mathrm{~W} / \mathrm{cm}^{2}$, the absorption lines are ac Stark-shifted. This corresponds to a decrease in absorbance at the position of the static lineshape. The spectral resolution ( $325 \mathrm{meV}$ at this energy, see previous section) prevents the observation of hyperbolic sidebands and the direct measurement of the temporal decay of these states ${ }^{2,55}$. However, the delay dependence of the pump-induced bleach of the absorption line can give an upper bound of the temporal resolution of our experiment. The changes in absorbance as a function of the SXR-NIR delay for the $2 \mathrm{p}_{1 / 2}^{-1} 3 \mathrm{~d}, 2 \mathrm{p}_{3 / 2}^{-1} 3 \mathrm{~d}$ and $2 \mathrm{p}_{3 / 2}^{-1} 4 \mathrm{~s}$ states are shown in Fig. $6 \mathrm{~b}-\mathrm{d}$, respectively. For these three states, the changes can be fit with a Gaussian function of $\sim 11 \mathrm{fs} \mathrm{fwhm}$. We note that in a similar experiment employing SXR and two-cycle $1700 \mathrm{~nm}$ pulses, Chew et al. observed sub-cycle oscillations that were attributed to a signature of tunnel ionization ${ }^{29}$. Due to averaging of the CEP, these oscillations are not resolved in our experiments. It would be interesting to study such processes in the future, as the calculated Keldysh parameter in our experiment is also in the tunneling regime but different than at $1700 \mathrm{~nm}$.

\title{
Discussion
}

The simultaneous production of millijoule-level sub-13 fs pulses at $1300 \mathrm{~nm}$ and sub- $5 \mathrm{fs}$ pulses at $800 \mathrm{~nm}$ with two parallel hollow-core fiber compressors has been demonstrated. The few-cycle SWIR pulses can produce a high-flux high-harmonic supercontinuum in the soft X-ray region, with $\sim 4.5 \times 10^{7}$ photons $/ \mathrm{s}$ in $1 \%$ bandwidth at $200 \mathrm{eV}$ and $\sim 1.4 \times 10^{6}$ photons/s in $1 \%$ bandwidth at $300 \mathrm{eV}$. This brightness allows measurement of absorption spectra in the gas phase in a few seconds, an order of magnitude improvement compared to existing SXR HHG 

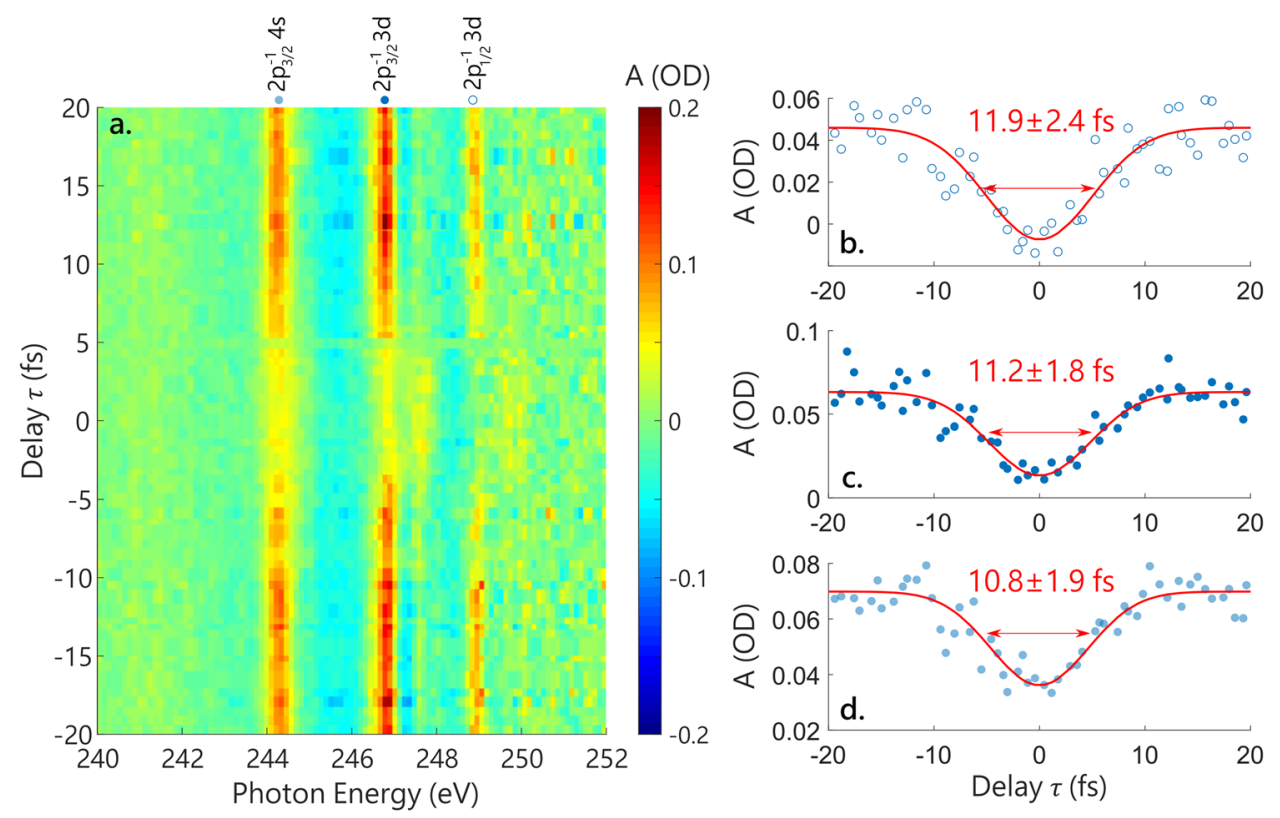

Figure 6. Transient absorption spectroscopy in the vincinity of the $\operatorname{Ar} \mathrm{L}_{2,3}$ edge with sub- 5 fs vis-NIR pulses. (a) Soft X-ray absorption spectra of argon as a function of the delay between the SXR and vis-NIR pulses. Negative delay values correspond to the SXR arriving first. At each time step the spectrum is acquired during a period of $15 \mathrm{~s}$, for a total measurement time of $38 \mathrm{~min}$. (b-d) Lineouts of (a) at the $2 \mathrm{p}_{1 / 2}^{-1} 3 \mathrm{~d}$ energy (b), $2 \mathrm{p}_{3 / 2}^{-1} 3 \mathrm{~d}$ energy (c) and $2 \mathrm{p}_{3 / 2}^{-1} 4 \mathrm{~s}$ energy $(\mathbf{d})$. The blue dots are the experimental data and the red line is a Gaussian fit, whose full width at half-maximum duration is indicated.

sources based on few-cycle driving pulses at longer wavelength ${ }^{22,29,48}$. The demonstration of increased photon flux using a short SWIR wavelength and a long HHG medium represents an important step towards the observation of attosecond dynamics in solution phase with transient absorption in the water window ${ }^{56}$. The dual-wavelength approach furthermore allows the strong-field excitation of molecules ${ }^{4,34,38}$ or single-photon pumping across a majority of semiconductor bandgaps $s^{8,9}$, including $2 \mathrm{D}$ semiconductors ${ }^{57}$. The broadband, few-cycle vis-NIR pulse could additionally be upconverted to few-fs tunable UV pulses ${ }^{58}$, opening new possibilities for the study of ultrafast excited-state dynamics in molecules ${ }^{59-61}$. A temporal resolution of $\sim 11 \mathrm{fs}$ is measured in a pump-probe experiment. This duration is already enough to consider the observation of ultrafast chemical dynamics at the C K-edge en $^{30,31,53,59}$. Improved CEP stability and feedback control over the delay will allow attosecond transient absorption experiments in the SXR in the future.

Received: 5 December 2019; Accepted: 9 March 2020;

Published online: 01 April 2020

\section{References}

1. Goulielmakis, E. et al. Real-time observation of valence electron motion. Nature 466, 739, https://doi.org/10.1038/nature09212 (2010).

2. Wang, H. et al. Attosecond time-resolved autoionization of argon. Physical Review Letters 105, 143002, https://doi.org/10.1103/ PhysRevLett.105.143002 (2010).

3. Ott, C. et al. Reconstruction and control of a time-dependent two-electron wave packet. Nature 516, 374, https://doi.org/10.1038/ nature14026 (2014)

4. Wei, Z. et al. Elucidating the origins of multimode vibrational coherences of polyatomic molecules induced by intense laser fields. Nature Communications 8, 735, https://doi.org/10.1038/s41467-017-00848-2 (2017)

5. Kobayashi, Y., Chang, K. F., Zeng, T., Neumark, D. M. \& Leone, S. R. Direct mapping of curve-crossing dynamics in IBr by attosecond transient absorption spectroscopy. Science 365, 79-83, https://doi.org/10.1126/science.aax0076 (2019).

6. Timmers, H. et al. Disentangling conical intersection and coherent molecular dynamics in methyl bromide with attosecond transient absorption spectroscopy. Nature Communications 10, 3133, https://doi.org/10.1038/s41467-019-10789-7 (2019).

7. Schultze, M. et al. Controlling dielectrics with the electric field of light. Nature 493, 75, https://doi.org/10.1038/nature11720 (2013).

8. Schultze, M. et al. Attosecond band-gap dynamics in silicon. Science 346, 1348-1352, https://doi.org/10.1126/science.1260311 (2014).

9. Zürch, M. et al. Direct and simultaneous observation of ultrafast electron and hole dynamics in germanium. Nature Communications 8, 15734, https://doi.org/10.1038/ncomms15734 (2017).

10. Schlaepfer, F. et al. Attosecond optical-field-enhanced carrier injection into the GaAs conduction band. Nature Physics 14, 560 (2018).

11. Geneaux, R., Marroux, H. J., Guggenmos, A., Neumark, D. M. \& Leone, S. R. Transient absorption spectroscopy using high harmonic generation: a review of ultrafast x-ray dynamics in molecules and solids. Philosophical Transactions of the Royal Society A 377, 20170463, https://doi.org/10.1098/rsta.2017.0463 (2019).

12. Ren, X. et al. Attosecond light sources in the water window. Journal of Optics 20, 023001, https://doi.org/10.1088/2040-8986/aaa394 (2018). 
13. Krause, J. L., Schafer, K. J. \& Kulander, K. C. High-order harmonic generation from atoms and ions in the high intensity regime. Physical Review Letters 68, 3535-3538, https://doi.org/10.1103/PhysRevLett.68.3535 (1992).

14. Spielmann, C. et al. Generation of coherent X-rays in the water window using 5-femtosecond laser pulses. Science 278, 661-664, https://doi.org/10.1126/science.278.5338.661 (1997).

15. Chang, Z., Rundquist, A., Wang, H., Murnane, M. M. \& Kapteyn, H. C. Generation of coherent soft X rays at $2.7 \mathrm{~nm}$ using high harmonics. Physical Review Letters 79, 2967-2970, https://doi.org/10.1103/PhysRevLett.79.2967 (1997).

16. Takahashi, E. J., Kanai, T., Ishikawa, K. L., Nabekawa, Y. \& Midorikawa, K. Coherent water window X ray by phase-matched highorder harmonic generation in neutral media. Physical Review Letters 101, 253901, https://doi.org/10.1103/PhysRevLett.101.253901 (2008).

17. Popmintchev, T. et al. Phase matching of high harmonic generation in the soft and hard $\mathrm{x}$-ray regions of the spectrum. Proceedings of the National Academy of Sciences 106, 10516-10521, https://doi.org/10.1073/pnas.0903748106 (2009).

18. Chen, M.-C. et al. Bright, coherent, ultrafast soft X-ray harmonics spanning the water window from a tabletop light source. Physical Review Letters 105, 173901, https://doi.org/10.1103/PhysRevLett.105.173901 (2010).

19. Popmintchev, T. et al. Bright coherent ultrahigh harmonics in the $\mathrm{keV} \mathrm{x}$-ray regime from mid-infrared femtosecond lasers. Science 336, 1287-1291 (2012)

20. Ishii, N. et al. Carrier-envelope phase-dependent high harmonic generation in the water window using few-cycle infrared pulses. Nature Communications 5, 3331, https://doi.org/10.1038/ncomms4331 (2014).

21. López-Martens, R. et al. Amplitude and phase control of attosecond light pulses. Physical Review Letters 94, 033001, https://doi. org/10.1103/PhysRevLett.94.033001 (2005).

22. Li, J. et al. 53-attosecond X-ray pulses reach the carbon K-edge. Nature Communications 8, 1-5, https://doi.org/10.1038/s41467-01700321-0 (2017).

23. Teichmann, S. M., Silva, F., Cousin, S. L., Hemmer, M. \& Biegert, J. 0.5-keV Soft X-ray attosecond continua. Nature Communications 7, 11493, https://doi.org/10.1038/ncomms11493 (2016).

24. Johnson, A. S. et al. High-flux soft $\mathrm{x}$-ray harmonic generation from ionization-shaped few-cycle laser pulses. Science Advances 4,https://doi.org/10.1126/sciadv.aar3761 (2018).

25. Baltuška, A., Fuji, T. \& Kobayashi, T. Controlling the carrier-envelope phase of ultrashort light pulses with optical parametric amplifiers. Physical Review Letters 88, 133901, https://doi.org/10.1103/PhysRevLett.88.133901 (2002).

26. Schmidt, B. E. et al. Compression of $1.8 \mu$ m laser pulses to sub two optical cycles with bulk material. Applied Physics Letters 96, 121109, https://doi.org/10.1063/1.3359458 (2010).

27. Tate, J. et al. Scaling of wave-packet dynamics in an intense midinfrared field. Physical Review Letters 98, 013901, https://doi. org/10.1103/PhysRevLett.98.013901 (2007).

28. Shiner, A. D. et al. Wavelength scaling of high harmonic generation efficiency. Physical Review Letters 103, 073902, https://doi. org/10.1103/PhysRevLett.103.073902 (2009).

29. Chew, A. et al. Attosecond transient absorption spectrum of argon at the $L_{23,}$ edge. Phys. Rev. A 97, 031407, https://doi.org/10.1103/ PhysRevA.97.031407 (2018).

30. Northey, T. et al. Ultrafast nonadiabatic dynamics probed by nitrogen K-edge absorption spectroscopy. Physical Chemistry Chemical Physics 22, 2667-2676, https://doi.org/10.1039/C9CP03019K (2020).

31. Tsuru, S. et al. Time-resolved near-edge x-ray absorption fine structure of pyrazine from electronic structure and nuclear wave packet dynamics simulations. The Journal of Chemical Physics 151, 124114, https://doi.org/10.1063/1.5115154 (2019).

32. Hua, W., Mukamel, S. \& Luo, Y. Transient x-ray absorption spectral fingerprints of the s1 dark state in uracil. The Journal of Physical Chemistry Letters, https://doi.org/10.1021/acs.jpclett.9b02692 (2019).

33. Buades, B. et al. Attosecond-resolved petahertz carrier motion in semi-metallic $\mathrm{TiS}_{2}$. arXiv preprint arXiv:1808.06493 (2018)

34. Saito, N. et al. Real-time observation of electronic, vibrational, and rotational dynamics in nitric oxide with attosecond soft $\mathrm{x}$-ray pulses at $400 \mathrm{eV}$. Optica 6, 1542-1546, https://doi.org/10.1364/OPTICA.6.001542 (2019).

35. Nisoli, M. et al. Compression of high-energy laser pulses below 5 fs. Optics Letters 22, 522-524, https://doi.org/10.1364/ OL.22.000522 (1997).

36. Giguère, M. et al. Pulse compression of submillijoule few-optical-cycle infrared laser pulses using chirped mirrors. Optics Letters 34, 1894-1896, https://doi.org/10.1364/OL.34.001894 (2009).

37. Mahieu, B. et al. Spatial quality improvement of a ti: Sapphire laser beam by modal filtering. Applied Physics B 118, 47-60, https:// doi.org/10.1007/s00340-014-5953-4 (2015).

38. Timmers, H. et al. Generating high-contrast, near single-cycle waveforms with third-order dispersion compensation. Optics Letters 42, 811-814, https://doi.org/10.1364/OL.42.000811 (2017).

39. Silva, F. et al. Simultaneous compression, characterization and phase stabilization of gw-level 1.4 cycle vis-nir femtosecond pulses using a single dispersion-scan setup. Optics Express 22, 10181-10191, https://doi.org/10.1364/OE.22.010181 (2014).

40. Ishii, N., Kaneshima, K., Kanai, T., Watanabe, S. \& Itatani, J. Generation of sub-two-cycle millijoule infrared pulses in an optical parametric chirped-pulse amplifier and their application to soft $\mathrm{x}$-ray absorption spectroscopy with high-flux high harmonics. Journal of Optics 20, 014003, https://doi.org/10.1088/2040-8986/aa99ed (2018).

41. Bor, Z. Distortion of femtosecond laser pulses in lenses and lens systems. Journal of Modern Optics 35, 1907-1918, https://doi. org/10.1080/713822325 (1988).

42. Sairanen, O.-P., Kivimäki, A., Nõmmiste, E., Aksela, H. \& Aksela, S. High-resolution pre-edge structure in the inner-shell ionization threshold region of rare gases Xe, Kr, and Ar. Phys. Rev. A 54, 2834-2839, https://doi.org/10.1103/PhysRevA.54.2834 (1996).

43. Constant, E. et al. Optimizing high harmonic generation in absorbing gases: Model and experiment. Physical Review Letters 82, 1668-1671, https://doi.org/10.1103/PhysRevLett.82.1668 (1999).

44. Sutherland, J. R. et al. High harmonic generation in a semi-infinite gas cell. Optics Express 12, 4430-4436, https://doi.org/10.1364/ OPEX.12.004430 (2004).

45. Baltuška, A. et al. Attosecond control of electronic processes by intense light fields. Nature 421, 611, https://doi.org/10.1038/ nature01414 (2003).

46. Cousin, S. L. et al. Attosecond streaking in the water window: a new regime of attosecond pulse characterization. Physical Review X 7, 041030, https://doi.org/10.1103/PhysRevX.7.041030 (2017).

47. Schibli, T. R. et al. Attosecond active synchronization of passively mode-locked lasers by balanced cross correlation. Optics Letters 28, 947-949, https://doi.org/10.1364/OL.28.000947 (2003).

48. Johnson, A. et al. Measurement of sulfur $\mathrm{L}_{2,3}$ and carbon $\mathrm{K}$ edge XANES in a polythiophene film using a high harmonic supercontinuum. Structural Dynamics 3, 062603, https://doi.org/10.1063/1.4964821 (2016).

49. Johnson, A. S. et al. Apparatus for soft $\mathrm{x}$-ray table-top high harmonic generation. Review of Scientific Instruments 89, 083110, https:// doi.org/10.1063/1.5041498 (2018).

50. Buades, B. et al. Dispersive soft x-ray absorption fine-structure spectroscopy in graphite with an attosecond pulse. Optica 5, 502-506, https://doi.org/10.1364/OPTICA.5.000502 (2018).

51. Popmintchev, D. et al. Near- and extended-edge x-ray-absorption fine-structure spectroscopy using ultrafast coherent high-order harmonic supercontinua. Physical Review Letters 120, 093002, https://doi.org/10.1103/PhysRevLett.120.093002 (2018).

52. Hudson, E. et al. High-resolution measurements of near-edge resonances in the core-level photoionization spectra of SF 6 . Phys. Rev. A 47, 361-373, https://doi.org/10.1103/PhysRevA.47.361 (1993). 
53. Pertot, Y. et al. Time-resolved $\mathrm{x}$-ray absorption spectroscopy with a water window high-harmonic source. Science 355, 264-267, https://doi.org/10.1126/science.aah6114 (2017).

54. Sham, T. K., Yang, B. X., Kirz, J. \& Tse, J. S. K-edge near-edge x-ray-absorption fine structure of oxygen- and carbon-containing molecules in the gas phase. Physical Review A 40, 652-669, https://doi.org/10.1103/PhysRevA.40.652 (1989).

55. Beck, A. R. et al. Attosecond transient absorption probing of electronic superpositions of bound states in neon: detection of quantum beats. New Journal of Physics 16, 113016, https://doi.org/10.1088/1367-2630/16/11/113016 (2014).

56. Kleine, C. et al. Soft X-ray absorption spectroscopy of aqueous solutions using a table-top femtosecond soft X-ray source. The Journal of Physical Chemistry Letters 10, 52-58, https://doi.org/10.1021/acs.jpclett.8b03420 (2019).

57. Manzeli, S., Ovchinnikov, D., Pasquier, D., Yazyev, O. V. \& Kis, A. 2D transition metal dichalcogenides. Nature Reviews Materials 2, 17033, https://doi.org/10.1038/natrevmats.2017.33 (2017).

58. Varillas, R. B. et al. Microjoule-level, tunable sub-10 fs UV pulses by broadband sum-frequency generation. Optics Letters 39, 3849-3852, https://doi.org/10.1364/OL.39.003849 (2014).

59. Attar, A. R. et al. Femtosecond $\mathrm{x}$-ray spectroscopy of an electrocyclic ring-opening reaction. Science 356, 54-59, https://doi. org/10.1126/science.aaj2198 (2017)

60. Bhattacherjee, A. et al. Photoinduced heterocyclic ring opening of furfural: Distinct open-chain product identification by ultrafast $\mathrm{x}$-ray transient absorption spectroscopy. Journal of the American Chemical Society 140, 12538-12544, https://doi.org/10.1021/ jacs.8b07155 (2018)

61. Schnorr, K. et al. Tracing the $267 \mathrm{~nm}$-induced radical formation in dimethyl disulfide using time-resolved $\mathrm{x}$-ray absorption spectroscopy. The Journal of Physical Chemistry Letters 10, 1382-1387, https://doi.org/10.1021/acs.jpclett.9b00159 (2019).

\section{Acknowledgements}

We acknowledge Profs. Roger Falcone, Eric Neuscamann, Feng Wang and Norman Yao for fruitful discussions. We thank Dr. Vincent Gruson and Dr. Stefan Haessler for helpful discussions about the pulse compression, and Dr. Romain Géneaux for comments on the manuscript. This work is supported by the W.M. Keck Foundation Grant No. 042982, the National Science Foundation through NSF MRI 1624322 and matching funds from the Lawrence Berkeley National Laboratory, the College of Chemistry, the Department of Physics, and the Vice Chancellor for Research at UC Berkeley, and the US Department of Energy, Office of Science, Office of Basic Energy Sciences, Chemical Sciences, Geosciences and Biosciences Dividsion under contract DE-AC0205CH11231, FWP no. CHPHYS01. L.B. acknowledges support from the Miller Institute for Basic Research in Science at UC Berkeley.

\section{Author contributions}

L.B. and A.D.R. contributed equally to this work. A.D.R., P.M.K., D.M.N. and S.R.L. designed the project. L.B., A.D.R. and S.G. performed the experiments. D.M.N. and S.R.L. supervised the project. L.B. and A.D.R. wrote the manuscript, with inputs from all the authors. All authors reviewed the manuscript.

\section{Competing interests}

The authors declare no competing interests.

\section{Additional information}

Correspondence and requests for materials should be addressed to S.R.L.

Reprints and permissions information is available at www.nature.com/reprints.

Publisher's note Springer Nature remains neutral with regard to jurisdictional claims in published maps and institutional affiliations.

(c) (i) Open Access This article is licensed under a Creative Commons Attribution 4.0 International (c) License, which permits use, sharing, adaptation, distribution and reproduction in any medium or format, as long as you give appropriate credit to the original author(s) and the source, provide a link to the Creative Commons license, and indicate if changes were made. The images or other third party material in this article are included in the article's Creative Commons license, unless indicated otherwise in a credit line to the material. If material is not included in the article's Creative Commons license and your intended use is not permitted by statutory regulation or exceeds the permitted use, you will need to obtain permission directly from the copyright holder. To view a copy of this license, visit http://creativecommons.org/licenses/by/4.0/.

(C) The Author(s) 2020 